\title{
Peripheral Venous Cannulation Associated Thrombophlebitis And Its Management
}

\author{
Shiana Singh, Amit Gupta, Prince Handa, Naman Aggarwal, Sweety Gupta, Vasantha C. Kalyani, \\ Chongtham Anil Kumar Singh, Deepak Rajput, Navin Kumar, and Manoj Gupta
}

\begin{abstract}
Introduction: Thrombophlebitis due to peripheral venous catheterization is one of the most common yet the most neglected iatrogenic complications in health care settings. If taken care of, the incidence of thrombophlebitis can be significantly reduced thus reducing the burden of its complications, patient's hospital stay and exploitation of human and economical resources.

Material and Methods: This observational study of thrombophlebitis after peripheral venous cannulation was carried out at a tertiary care centre. 761 patients admitted in different clinical departments were subjected to peripheral venous catheterization for various therapeutic treatment.

Results: 100 patients developed thrombophlebitis out of 761 patients. Majority of the patients were managed by conservative methods, only one patient was managed by incisional pus drainage.

Conclusion: From this study we conclude that the incidence of thrombophlebitis in our hospital setting was around $10 \%$. Taking aseptic precautions, proper cannula care and changing the cannula timely can significantly reduce the incidence. Most of the cases can be managed non-operatively by giving medications according to symptoms.
\end{abstract}

Index Terms - Intravenous Cannulation, peripheral, thrombophlebitis.

\section{INTRODUCTION}

Peripheral venous cannulation has revolutionized the practice of drug administration. IV catheters allow infusion of medications, hydration fluids, blood products and nutritional supplements. Along with the benefits, a number of potential complications are associated with IV cannula, ranging from minor pain to severe thrombophlebitis and catheter related infections.

Infection control plays a vital role in health care practices but unfortunately it is often the under-recognized and under supported part of the health care system. It is one of the oldest problems in the history of medical practice and currently it is posing new challenges to medical profession. According to WHO, 1 in every 10 patients get an infection while receiving health care. Most of the patients admitted in the hospital are

Published on June 6, 2020.

S. Singh, All India Institute of Medical Sciences Rishikesh Uttarakhand, India.

A. Gupta, All India Institute of Medical Sciences Rishikesh Uttarakhand, India.

(corresponding e-mail: dramit2411@gmail.com)

P. Handa, All India Institute of Medical Sciences Rishikesh Uttarakhand, India.

N. Aggarwal, All India Institute of Medical Sciences Rishikesh Uttarakhand, India.

S. Gupta, All India Institute of Medical Sciences Rishikesh Uttarakhand, India. given some medications intravenously by inserting a catheter [1]. Intravenous catheters cause trauma to the vascular endothelium and inflammation. 70-80\% of thrombotic events occurring in the veins of upper extremity are due to intra venous catheter and the remaining are due to mechanical compression by anatomical abnormalities [2] - [4]. Many studies have concluded that thrombophlebitis is one of the most frequently associated complications of peripheral vein infusion, occurring in up to a fourth of hospitalized patients receiving intravenous therapy via veins of forearm and hand be it due to catheter material, infusate or microorganisms. Because of localized stasis and prostaglandin, mediated coagulation cascade there is thrombus formation [5]. The thrombus formation can lead to various complications that were studied in this study.

Because peripheral venous catheterization can lead to severe complications, basic medical research is significant in this field and not much information is available on this topic especially in Indian health care system. Globally, many strategies have been adopted to prevent/minimize the complications related to peripheral venous catheterization. However, in Indian scenario, not much breakthrough has been achieved so far. This study undertakes to fill this gap.

\section{MAterials AND Methods}

This observational study was done between June 2018 to August 2018. 761 patients admitted in different clinical departments of a tertiary care center were subjected to peripheral venous catheterization for various therapeutic treatment. After informed consent patients were catheterized after taking all the septic precautions. All the inserted IV catheters were examined daily to determine the complications associated with the catheter, if any. In addition, the routine replacement of catheter was documented in order. Patients were followed up daily to get the above details.

Thrombophlebitis in patients was graded according to the phlebitis scale given by the Infusion Nurses Society (2006) as follows:

- Grade 0: No symptoms;

V. C. Kalyani, All India Institute of Medical Sciences Rishikesh Uttarakhand, India.

C. A. K. Singh, AIIMS Rishikesh, India.

D. Rajput, All India Institute of Medical Sciences Rishikesh Uttarakhand, India.

N. Kumar, All India Institute of Medical Sciences Rishikesh Uttarakhand, India.

M. Gupta, All India Institute of Medical Sciences Rishikesh Uttarakhand, India. 
- Grade 1: Erythema at access site with or without pain;

- Grade 2: Pain at the access site with erythema and/or oedema;

- Grade 3: Pain at the access site with erythema and/or oedema, streak formation, palpable venous cord;

- Grade 4: Pain at the access site with erythema and/or oedema, streak formation, palpable venous cord greater than one inch in length and purulent drainage.

Patient performa was prepared and filled by asking questions and examining the patient by the medical and nursing team. The performa included the patient, date of initial peripheral catheterization, date of re-catheterization, cause of re-catheterization, patient's complaints, examination findings, grading of thrombophlebitis and treatment given.

Institutional ethical committee approval was taken via Letter No AIIMS/IEC/18/311. Date: 11/07/2018.

\section{RESULTS}

A total of 100 cases out of 761 developed thrombophlebitis and were included in this study. 72 patients in department of Surgery, 23 patients of department of Medicine developed thrombophlebitis, remaining 5 patients were taken from other departments (Radiation Oncology). Out of the cases, male: female ratio was 36:64, mean age was 48.8 years. Patient characteristics are shown in (Table I).

TABLE I: CHARACTERISTICS OF STUDY PARTICIPANTS

\begin{tabular}{lc}
\hline \hline \multicolumn{1}{c}{ Characteristic } & $\mathrm{N}(\%)$ \\
\hline Male & $36(36 \%)$ \\
Female & $64(64 \%)$ \\
\hline - Age (Mean) in years & $48.8(20-63$ years) \\
\hline Cannula (Gauge) & $32(32 \%)$ \\
- 18G & $66(66 \%)$ \\
- 20G & $02(02 \%)$ \\
\hline Total duration of cannulation (Mean) & $4.05(1-6)$ \\
\hline - Cannula per patient (Average) in days & 1.5 \\
\hline Infusates administered & \\
• Intravenous Fluids & $22(22 \%)$ \\
• Medication & $23(23 \%)$ \\
- Intravenous fluid + Medication & $55(55 \%)$ \\
\hline \hline
\end{tabular}

$66 \%$ of patients developed thrombophlebitis with $20 \mathrm{G}$ cannula and only $2 \%$ of patients with $22 \mathrm{G}$ cannula developed thrombophlebitis. Mean time to develop thrombophlebitis was 48 hours. It was observed that occurrence of thrombophlebitis was more common in patients who had cannulation for more than four days. 54 patients developed grade 2, 19 grade 3 and only 1 patient had grade 4 thrombophlebitis that required surgical intervention. 55\% patients had received both intravenous fluids and medication and developed thrombophlebitis. Obese and diabetic patients had tendency for occurrence of phlebitis. Grading was done according to scale given by the Infusion Nurses Society (2006) and the results are shown in Fig. 1. 2.54\% of patients had grade 2 and only $1 \%$ had grade 4 phlebitis. In the management of thrombophlebitis, the first and the most important step is to remove the cannula and insert a new cannula at some other site. In mild cases, it resolves by itself after removal of the cannula but in severe cases, medication is required. Patients were prescribed anti-inflammatory, anti- pyretic and topical (Benzyl Nicotinate and Heparin, Magnesium Sulphate and Sulphacetamide ointment) medications according to the severity. In severe swelling limb elevation was given and antibiotics were prescribed in case of infection by various micro-organisms. Incisional pus drainage was also done. In the cases studied in this study, cannula was removed in all the cases and after that, symptomatic management was done (Fig. 1).

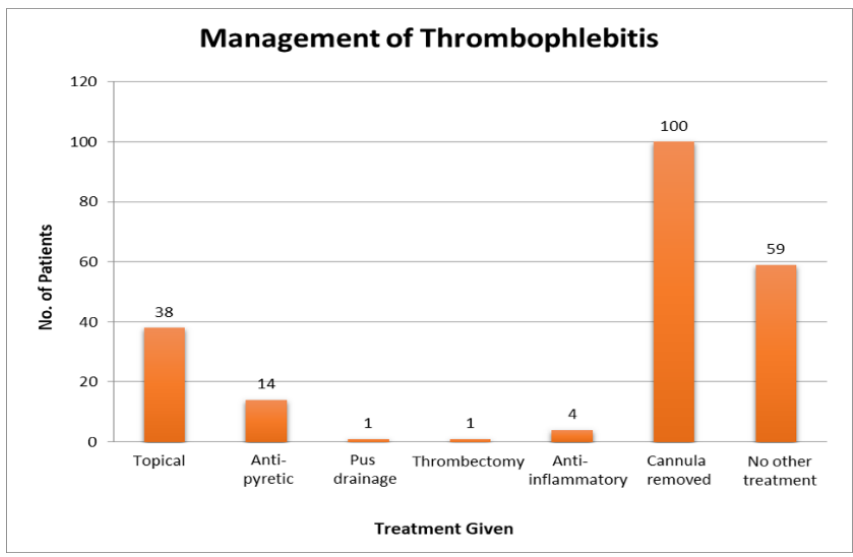

Fig. 1. Bar graph showing treatment given to patients having thrombophlebitis

\section{DISCUSSION}

The placement of intravascular cannula is one of the most common in patient procedures done in hospital setting. Although there are many benefits of IV therapy, these intravascular devices are related with widespread range of iatrogenic complications ranging from phlebitis to sepsis to death leading to prolonged hospitalization and increased health care costs. Phlebitis is defined as inflammation of vein and pathogenetic mechanism include mechanical, chemical or bacterial processes. Phlebitis manifests as following symptoms as pain, oedema, hardening and hyperaemia. If it worsens it can lead formation of palpable fibrous cord of the vein. There may also be purulent discharge from cannulation site. Rate of complication in the present study was equivalent to that in other studies. Predilection of patients to develop phlebitis depends on insertion method, anatomical location of cannulation site, size and type of catheter, number of attempts for insertion, catheters placed for more than 72 hours, severity of disease and pre-existing infections, irritant drugs eg chemotherapeutic agents and concentration of infusion [6], [7]. In the present study time to develop thrombophlebitis was similar of 48 hours as that mentioned in [8]. Apart from complications of pain, sepsis, cardiovascular effects phlebitis can lead to prolonged hospitalization and increased medical costs. Literature support skill in insertion, full asepsis and training level of nurses as important risk factors for the development of phlebitis. In our study one patient required incision and drainage.

\section{CONClusion}

Thrombophlebitis is less reported and reviewed topic but needs to be addressed by various prospective trials. In our present study we studied incidence of thrombophlebitis at our institute and risk factors for occurrence. It can be reduced by 
teaching cannulation technique to ward nurses, paramedics and doctors on simulated models. Measures such as aseptic precautions and use of gloves, nursing training and use of simulators for cannulation techniques play an important role in decreasing the incidence of thrombophlebitis.

\section{REFERENCES}

[1] H. Decousus, I. Quere, E. Presles, et al. "Superficial venous thrombosis and venous thromboembolism: a large, prospective epidemiologic study", Annals of internal medicine, vol. 152, pp. 218-224, 2010.

[2] M. Di Nisio, I. M. Wichers, S. Middeldorp, "Treatment for superficial thrombophlebitis of the leg", Cochrane Database Syst Rev., vol 4:CD004982, 2013.

[3] C. M. Rickard, G. Ray-Barruel, "Peripheral intravenous catheter assessment: beyond phlebitis", Lancet Haematol, vol. 4, pp. 402-403, 2017.

[4] K. Wong, A. Cooper, J. Brown, et al. "The prevalence of peripheral intravenous cannulae and pattern of use: A point prevalence in a private hospital setting", J ClinNurs, vol. 27(1-2), pp. 363-367, 2018.

[5] L. Zhang, S. Cao, N. Marsh, et al. "Infection risks associated with peripheral vascular catheters", J Infect Prev, vol. 17, pp. 207-213, 2016.

[6] M. D. Hagerstown, J. P. Lippincott, "Infusion Nurses Society", Infusion Nursing Standards of Practice, 2006.

[7] N. P. O'Grady, M. Alexander, L. A. Burns, et al. "Guidelines for the prevention of intravascular catheter-related infections", Clinical Infectious Diseases, vol. 52, pp. 162-193, 2011.

[8] M. T. Tork Torabi, M. Namnabati, Z. Allameh, S. Talakoub, "Vancomycin infusion methods on phlebitis prevention in children", Iranian J Nursing Midwifery Res, vol. 24, pp. 432-436, 2019. 